\title{
A Quantum-Like Approach to the Interaction of Relativistic Charged Particle Beams with Plasmas
}

\author{
R. Fedele, P.K. Shukla* and V.G. Vaccaro \\ Dipartimento di Scienze Fisiche, Università di Napoli "Federico II" and INFN Sezione di Napoli, \\ Mostra D'Oltremare Pad. 20,80125 Napoli, Italy \\ * Institut fïr Theoretische Physik IV, Ruhr-Universität Bochum, 44780 Bochum, Germany
}

\begin{abstract}
It is shown that particle beam dynamics can be described in terms of a Schrödingerlike equation for a complex function, the so- called beam wave function (BWF), whose squared modulus yields the density distribution of the particles in the configuration space. The role of Planck's constant is here played by the beam emittance (transverse or longitudinal). In this framework, we describe the interaction of a relativistic charged particle beam with an unmagnetized, collisionless plasma in terms of nonlinear Schrödinger-like equations fully similar to the ones are describing the nonlinear electromagnetic beam-plasma interaction. This way, both longitudinal and transverse particle dynamics in plasmas can be naturally described, and new insights are obtained readily.
\end{abstract}

\section{Introduction}

Relativistic charged particle beams are imployed [1] for generating intense electron plasma waves or wakefields. When the phase velocity of the latter is close to the speed of light, the plasma wave can trap a nearly relativistic electron and accelerate it to extremely high energies. The idea of this novel plasma-based charged particle acceleration scheme has been verified experimentally [2].

It is well known that interaction of relativistic charged particle beams of finite extent with the background plasma can give rise to the two-stream instability, the self-bunching, the self-focusing (self-defocusing), as well as the beam filamentation and the self-pinching.

Furthermore, the transverse (longitudinal) dynamics of a charged particle beam has been recently described in terms of a quantum-like model, the so-called Thermal Wave Model (TWM) [3]. According to this model the beam transport is described in terms of a complex function, the beam wave function (BWF), whose squared modulus gives the transverse (longitudinal) density profile. In TWM the beam wave function is assumed to satisfy a Schrödinger-like equation in which Planck's constant is replaced by the transverse (longitudinal) emittance. This equation is in general written for a potential which accounts for the total interaction between the beam and the sourrondings. In particular, in an accelerating machine the potential accounts for both multipole-like terms, not 
depending on the particle distribution but assigned in terms of the machine parameters, and collective terms which depend on the particle distribution (self- interaction). This way, the Schrödinger-like equation in TWM can be in general non linear [4]-[7].

In transverse dynamics, TWM has been successfully applied to a number of linear and nonlinear problem. In particular, it has been capable of both reproducing the main results of Gaussian particle beam optics and dynamics for a quadrupole-like device [3], and estimating the luminosity in final focusing stages of linear colliders in the presence of small sextupole- and octupole-like deviations [8].

For the case of transverse dynamics in quadrupole-like devices with small sextupole and octupole deviations, comparisons between TWM predictions and tracking code simulations showed a very satisfactory agreements [9],[10]. The analysis was performed in both configuration and momentum spaces.

Recently TWM has been also used for describing longitudinal dynamics of an electron bunch in a circular accelerating machine by tacking into account a radio-frequency potential well and both radiation damping and quantum excitation [11]. This way, the correct r.m.s. emittance scaling law was, in particular, found.

In this paper we present a first review concerning with the applications of TWM to the interaction of a relativistic charged particle beam with an unmagnetized, collisionless plasma.

In section 2, we give a brief presentation of TWM for the dynamics of a charged particle beam travelling in a plasma, whilst in section 3 we split our analysis in two parts: transverse and longitudinal beam dynamics. In subsection 3.1, we show that the self-interaction involved in the transverse dynamics of a relativistic electron (positron) beam can be correctly described by TWM in cold, unmagnetized plasmas [4]. In this case the main results of Weibel instability (filamentation) and Bennett's self-pinching, are recovered. In addition, the principle of the plasma wake-fields-based lens can be straightforwardly derived. In subsection 3.2 , we show that TWM can be applied to the longitudinal dynamics of a charged particle beam in plasma-based accelerators [7]. Fynally, section 4 contains conclusions and remarks.

\section{Formulation of the Problem}

Let us consider a cylindrically-symmetric relativistic charged particle beam travelling with velocity $\beta c(\beta \approx 1)$ and transverse (longitudinal) emittance $\epsilon$. We suppose that, at $s=0(s \equiv c t)$, the beam enters a semi- infinite plasma slab where in general could be already present a plasma wave travelling along the same propagation direction of the particle beam with phase velocity $\beta_{p h} c$. We suppose that the electric field associated with the plasma wave has a transverse (longitudinal) profile. If the beam is injected in the plasma in such a way to be in phase-locking with the wave $\left(\beta=\beta_{p h}\right)$, the beam particle execute transverse (longitudinal) motion with respect to the synchronous particle which is, by definition, the particle remaining at rest with respect to the wave during the motion through the plasma. Note that the potential associated to the wave play the role of an external potential acting on the beam.

In the framework of the TWM, the transverse (longitudinal) beam dynamics is governed 
by the following Schrödinger-like equation [3] ([6]),

$$
i \epsilon \frac{\partial \Psi}{\partial s}=-\frac{\epsilon^{2}}{2} \nabla_{x}^{2} \Psi+U(x, s) \Psi
$$

where $U(x, s)$ is a dimensionless potential energy normalized with respect to $m_{0} \gamma \beta^{2} c^{2}\left(m_{0}\right.$ and $\gamma$ being the particle rest mass and the relativistic factor $\left.\left(1-\beta^{2}\right)^{-1 / 2}\right), x$ stands for the set of transverse (longitudinal) coordinates, and $\nabla_{x}^{2}$ denotes the lapacian with respect to this set of coordinates. $U$ accounts for the external potential energy $U_{w}(x, s)$ as well as the self-interaction potential (wake-potential energy) $U_{s}(x, s)$, namely

$$
U(x, s)=U_{w}(x, s)+U_{s}(x, s) .
$$

Here, $|\Psi(x, s)|^{2}$ is interpreted as the transverse (longitudinal) density profile. Thus, provided that the following normalization condition

$$
\int_{-\infty}^{\infty}|\Psi(x, s)|^{2} d x<\infty \quad
$$

is satisfied, the transverse (longitudinal) number density $\rho_{b}(x, s)$ can be written as

$$
\rho_{b}(x, s) \equiv n_{b}|\Psi(x, s)|^{2}
$$

where $n_{b}$ is a positive constant.

We assume that for $s=0$ the beam has a Gaussian density distribution of r.m.s. $\sigma_{0}$, which corresponds to an initial Gaussian BWF associated with (1).

It is well-known that when a charged particle bunch of length $\sigma$, much greater than the plasma wavelength $\lambda_{p}$, is injected in an overdense, cold plasma, the following local charge-neutrality condition

$$
e n_{1}(x, s) \approx q \rho_{b}(x, s)
$$

is satisfied [1], where $e$ is the absolute value of the electron charge, $q$ is the charge of the particles of the beam, and $n_{1}$ is the plasma number density perturbation $\left(n_{1}<<n_{p}, n_{p}\right.$ being the unperturbed plasma number density). In addition, according to the standard description [1], the plasma wake-potential energy $U_{s}$ is related to the plasma density perturbation $n_{1}$ by (cylindrical symmetry):

$$
\left(\nabla_{\perp}^{2}-k_{p}^{2}\right) U_{s}=\frac{4 \pi q^{2} \rho_{b}(x, s)}{m_{0} \gamma \beta^{2} c^{2}} \approx \frac{4 \pi q^{2} n_{b}|\Psi(x, s)|^{2}}{m_{0} \gamma c^{2}},
$$

where $k_{p} \equiv \frac{2 \pi}{\lambda_{p}}=\frac{4 \pi e^{2} n_{p}}{m_{0} c^{2}}$ is the plasma wave number. Eq.s (1) with (2) and (6) constitute a system of coupled equations describing, in the framework of TWM, the self-consistent interaction of a relativistic charged particle beam with the plasma where a plasma wave is in general also present.

\section{Charged Particle Beam Dynamics}

In this section we consider two limiting cases of this description. First, we analyse a purely transverse beam dynamics, by assuming negligible any longitudinal dynamics. Then, we consider the case of a transversally flat bunch for which the transverse dynamic can be neglected. 


\subsection{Purely Transverse Beam Dynamics}

By assuming a cylindrical symmetry, the transverse coordinate $x$, introduce above, must be replaced here by the radial cylindrical coordinate $r$, while $s$ can be identified with the coordinate $z$ along the propagation direction. Under these hypothesis, the following virial equation and energy-variation equation hold (for more details see Ref.[3]:

$$
\begin{gathered}
\frac{d^{2} R^{2}(z)}{d z^{2}}=4 \mathcal{E}-4 \mathcal{V}-\frac{4 \pi}{\mathcal{N}^{2}} \int_{0}^{\infty}\left(r \frac{\partial U}{\partial r}\right)|\Psi|^{2} r d r \\
\frac{d \mathcal{E}}{d z}=<\frac{\partial U}{\partial z}>=\frac{2 \pi}{\mathcal{N}^{2}} \int_{0}^{\infty}\left(\frac{\partial U}{\partial z}\right)|\Psi|^{2} r d r .
\end{gathered}
$$

$\left(R(z) \equiv\left[\left(2 \pi / \mathcal{N}^{2}\right) \int_{0}^{\infty} r d r r^{2}|\Psi|^{2}\right]^{1 / 2}\right.$ and $\mathcal{E}(z) \equiv\left(2 \pi / \mathcal{N}^{2}\right) \int_{0}^{\infty} r d r\left[\left(\epsilon^{2} / 2\right)\left|\nabla_{\perp} \Psi\right|^{2}+U|\Psi|^{2}\right]$ are the effective beam radius and the averaged total beam energy associated to the transverse motion, respectively). Let us consider $U_{w}$ turned off.

In order to illustrate how the equations (1) and (6) self-consistently describe the beam self-focusing, we consider two special cases.

\section{(A). SELF-FOCUSING}

First, we assume that the beam spot size is larger than the plasma wavelength (viz. $k_{p} R \gg>1$ or $\left.k_{p}^{2} \gg\left|\nabla_{\perp}\right|^{2}\right)$. Consequently, we obtain the following cubic nonlinear Schrödinger equation :

$$
i \epsilon \frac{\partial}{\partial z} \Psi=-\frac{\epsilon^{2}}{2} \nabla_{\perp}^{2} \Psi-\frac{n_{b}}{n_{p} \gamma}|\Psi|^{2} \Psi
$$

Equation (9) is similar in structure to the equation which describes the self-focusing of a coherent electromagnetic beam in a nonlinear medium. We easily find that in this case the averaged total energy $\mathcal{E}$ associated with the beam is not conserved. In fact, the charged particle beam interacting with the plasma is an open system. However, by using (8) it can be proved that the quantity

$$
\mathcal{A} \equiv \mathcal{E}+\frac{1}{2}\left[\frac{2 \pi}{\mathcal{N}^{2}} \frac{n_{b}}{n_{p} \gamma} \int_{0}^{\infty}|\Psi|^{4} r d r\right]
$$

is conserved, namely $d \mathcal{A} / d z=0$, and, consequently, by using the virial equation we get $d^{2} R^{2} / d z^{2}=4 \mathcal{A}=$ const., which can be immediately solved with the initial conditions $R_{0}=R(z=0)$ and $(d R / d z)_{z=0}=0$. We find $R^{2}(z)=R_{0}^{2}+2 \mathcal{A} z^{2}$. The latter shows that $\mathcal{A}$ is negative (positive) if $\left(2 \pi / \mathcal{N}^{2}\right)\left(\epsilon^{2} / 2\right) \int_{0}^{\infty}\left|\nabla_{\perp} \Psi\right|^{2} r d r$ (thermal energy) is smaller (larger) than half of the self energy, $\left(2 \pi / \mathcal{N}^{2}\right)\left(n_{b} / n_{p} \gamma\right) \int_{0}^{\infty}|\Psi|^{4} r d r$. Consequently, the charged particle beam would focus (defocus), as is evident from the equation for the beam caustic. A stationary solution also occurs when the thermal energy is exactly balanced by half of the self energy $(\mathcal{A}=0)$. It corresponds to a stabilization of the beam at the initial radius, which is analogous to the Bennett pinch equilibrium [12],[13]. A simple criterion for finding the threshold of the self-focusing can now be established. If we assume that the beam has a finite temperature $T$ (energy units) and an initial Gaussian profile, we find

$$
\mathcal{A} \approx \frac{1}{2}\left[\frac{\epsilon^{2}}{R_{0}^{2}}-\frac{1}{2} \frac{n_{b}}{n_{p} \gamma}\right] .
$$


So that, by imposing $\mathcal{A} \approx 0$, we immediately obtain the threshold condition

$$
\frac{\epsilon^{2}}{R_{0}^{2}} \approx \frac{1}{2} \frac{n_{b}}{n_{p} \gamma}
$$

which, since $\epsilon^{2} / R_{0}^{2}=T /\left(m_{0} \gamma \beta^{2} c^{2}\right) \approx T /\left(m_{0} \gamma c^{2}\right)$, becomes:

$$
\beta_{\perp} \equiv \frac{v_{t h}}{c} \approx 0.7\left(\frac{n_{b}}{n_{p} \gamma}\right)^{1 / 2}
$$

where $v_{t h}=\left(T / m_{0} \gamma\right)$ is the electron (positron) thermal velocity. Equation (13) gives the threshold for the Weibel (or the filamentation) instability [12].

\section{(B). ENVELOPE EQUATION}

It is interesting observing that by putting a non self-consistent aberrationless solution of (9), of Gaussian form $\Psi=\exp \left(-r^{2} / R^{2}\right)$, in the virial equation (7), we find the following envelope equation :

$$
\frac{d^{2} R}{d z^{2}}+\frac{2 n_{b} /\left(n_{p} \gamma\right)}{R}-\frac{\epsilon^{2}}{R^{3}}=0
$$

which takes into account the self-force; it is similar to the well known envelope equation which is valid for fully neutralized relativistic beam [14].

\section{(C). SELF-PINCHING}

Next, we consider the limit in which the beam spot size is smaller than the plasma wavelength (viz $k_{p} R<<1$ or $k_{P}^{2}<<\left|\nabla_{\perp}\right|^{2}$ )). For this case, the virial equation (7) becomes [4]:

$$
\frac{d^{2} R}{d z^{2}}=\frac{\epsilon^{2}}{R^{3}}-\frac{2 K}{R}<\int_{0}^{r}\left|\Psi\left(r^{\prime}, z\right)\right|^{2} r^{\prime} d r^{\prime}>
$$

where $K \equiv 2 \pi e^{2} n_{b} /\left(m_{0} \gamma \beta^{2} c^{2}\right)$ is the focusing strength. By observing that $d^{2} R^{2} / d z^{2}=$ $2\left[R d^{2} R / d z^{2}+(d R / d z)^{2}\right]$, Eq. (15) with the initial conditions $R_{0}=R(z=0)$ and $(d R / d z)_{z=0}=0$ allows us to establish the equilibrium condition $\left(d^{2} R / d z^{2}=0\right)$, which, for an initial Gaussian profile gives us:

$$
\frac{\epsilon^{2}}{R_{0}^{2}}=\frac{1}{2} K R_{0}^{2}
$$

This is the well known Bennett self-pinch equilibrium condition [13]. By introducing the definition of the beam current $I=e N \beta c / \sigma_{z} \approx e N c / \sigma_{z}$ and using the transverse beam emittance definition in terms of the transverse temperature $T$, Eq.(16) gives the well known Bennett pinch relation [13] (c.g.s. units):

$$
\frac{I^{2}}{c^{2}}=N_{z} T
$$

where $N_{z}=N / \sigma_{z}$ estimates the number of particles per unit longitudinal beam length.

(D). OVERDENSE PLASMA LENS 
We observe that in the last limiting case an aberrationless solution of (15) is also possible (we have again $\Psi=e^{-r^{2} / R^{2}}$ ). So, in the limit in which $\left.\int_{0}^{r} \Psi\left(r^{\prime}, z\right)\right|^{2} r^{\prime} d r^{\prime} \approx r^{2} / 2$ $(r<<R)$, we recover the well known envelope equation:

$$
\frac{d^{2} R}{d z^{2}}+K R-\frac{\epsilon^{2}}{R^{3}}=0
$$

which has been used to describe the optics of an overdense plasma lens in linear regime for the final focus in linear colliders [15].

\subsection{Purely Longitudinal Dynamics}

Let us consider the motion of charged particle of charge $q>0$ with velocity $\beta c(\beta \approx 1)$ along $x$-direction (here, the longitudinal direction) in a cold, unmagnetized plasma where a plasma wave is present. We assume that the electric field associated with the plasma wave, with phase velocity $v_{\phi} \approx c$ is given by:

$$
\vec{E}_{p 0}=\hat{x} E_{p 0} \sin \phi(t)
$$

where $\hat{x}$ is the unitary vector along $x$-axis. The particle will be accelerated or decelerated by the electric field, depending on the initial relative phase between particle and wave. In general, this interaction causes the particle to slip in phase relative to the plasma wave. However, there is a particular phase $\phi_{s}$, called synchronous phase, for which the particle does not change its energy and remains locked in phase with the wave. By following the standard treatment of the single particle motion well known in particle accelerators [16], [17], we introduce a phase displacement $\tilde{\phi}$ with respect to the synchronous one, so that:

$$
\phi=\tilde{\phi}+\phi_{s} \equiv k_{p} x+\phi_{s},
$$

where $k_{p}$ is the plasma wave number $\left(k_{p} \equiv \omega_{p} / c, \omega_{p}\right.$ being the plasma frequency) which in the following is supposed to be a constant and $x$ is the longitudinal particle displacement with respect to the synchronous position. By denoting with $\mathcal{P} \equiv \frac{\Delta E}{E_{0}}=\frac{E-E_{0}}{E_{0}}\left(E_{0}=m_{0} \gamma c^{2}\right.$ being the synchronous particle energy), we get:

$$
\begin{gathered}
\frac{d x}{d s}=\frac{1}{\gamma^{2}} \mathcal{P} \\
\frac{d \mathcal{P}}{d s}=\frac{q E_{p 0}}{m_{0} \gamma c^{2}}\left[\sin \left(k_{p} x+\phi_{s}\right)-\sin \phi_{s}\right]
\end{gathered}
$$

where $s=c t, \gamma=\left(1-\beta^{2}\right)^{-1 / 2}$. It is easy to prove that (21) and (22) can be obtained from the following dimensionless hamiltonian:

$$
H=\frac{\mathcal{P}^{2}}{2 \gamma^{2}}+U_{w}
$$

where

$$
U_{w} \equiv \frac{q E_{p 0}}{m_{0} \gamma c^{2} k_{p}}\left[\left(k_{p} x-\sin k_{p} x\right) \sin \phi_{s}+\cos k_{p} x \cos \phi_{s}\right]
$$

(plasma wave potential well). 
For very small phase displacement, i.e. $|\tilde{\phi}|<<1$ (linear case), (24) becomes:

$$
U_{w} \approx \frac{\gamma^{2}}{2} K_{p} x^{2}+\text { const }
$$

where

$$
K_{p} \equiv-\frac{q E_{p 0} k_{p} \cos \phi_{s}}{m_{0} \gamma^{3} c^{2}}
$$

Note that, since we are concerning with non-modulated plasma wave number (usually in conventional accelerating machines the radio waves are frequency-modulated), it is necessary but not sufficient that $\phi_{s}$ satisfies the condition $\sin \phi_{s}=0$, namely $\phi_{s}=n \pi$, with $n$ integer. Thus, if $E_{p 0}>0$, a phase-stability criterion can be established from (26) for which $\cos \phi_{s}<0\left(\cos \phi_{s}>0\right)$ corresponds to stability (instability). Consequently, for getting synchronous phase it is sufficient to choose

$$
\phi_{s}=(2 n+1) \pi \text {. }
$$

In order to describe the particle motion in the plasma wave.in the presence of the self-interaction given by the plasma wake field, we assign the following dimensionless hamiltonian:

$$
H=\frac{\mathcal{P}^{2}}{2 \gamma^{2}}+U_{w}+U_{s}
$$

Hamilton's equations become now the following:

$$
\begin{gathered}
\frac{d x}{d s}=\frac{\partial H}{\partial \mathcal{P}}=\frac{1}{\gamma^{2}} \mathcal{P} \\
\frac{d \mathcal{P}}{d s}=-\frac{\partial H}{\partial x}=\frac{q E_{p 0}}{m_{0} \gamma c^{2}}\left[\sin \left(k_{p} x+\phi_{s}\right)-\sin \phi_{s}\right]+\frac{q^{2}}{e^{2} \gamma n_{p}} \frac{\partial \rho_{b}}{\partial x},
\end{gathered}
$$

where (24) and (6) in the case of transversally-flat beam have been used. Eq. (29) and (30) are fully similar to the motion equations which hold in particle accelerators for longitudinal charged particle dynamics when the interaction between the bunch and the surrounding medium is produced by both a $\mathrm{RF}$ cavity and a purely reactive longitudinal coupling impedance [18]. Consequently, within the framework of TWM, to describe the longitudinal dynamics the following Schrödinger-like equation can be assumed

$$
i \epsilon \frac{\partial \Psi}{\partial s}=\hat{H} \Psi
$$

where $\hat{H}=\frac{\hat{p}^{2}}{2 \gamma^{2}}+\left(U_{w}+U_{s}\right) \equiv \frac{\hat{\mathfrak{p}}^{2}}{2 \gamma^{2}}+U$, namely:

$$
i \epsilon \frac{\partial \Psi}{\partial s}=-\frac{\epsilon^{2}}{2 \gamma^{2}} \frac{\partial^{2} \Psi}{\partial x^{2}}+\left(U_{w}+U_{s}\right) \Psi
$$

For the linearized plasma wave potential well (see Eq.(25)) and by using (4), (32) becomes:

$$
i \tilde{\epsilon} \frac{\partial \Psi}{\partial s}=-\frac{\tilde{\epsilon}^{2}}{2} \frac{\partial^{2} \Psi}{\partial x^{2}}+\frac{1}{2} K_{p} x^{2} \Psi-\frac{q^{2}}{e^{2}} \frac{n_{b}}{n_{p} \gamma^{3}}|\Psi|^{2} \Psi
$$


where $\tilde{\epsilon} \equiv \epsilon / \gamma^{2}$. This wave equation, given for the present problem within the framework of the thermal wave model for particle dynamics, is a modified form of the well known nonlinear Schrödinger equation (NLS) [19] describing a number of very important nonlinear phenomena in physics [19],[20]. In particular, an analogous form of (33) seems to govern the nonlinear longitudinal dynamics in particle accelerators when both RF cavities and a reactive longitudinal coupling impedance are taken into account, as it has been recently pointed out [6].

\section{(A). SYNCHROTRON-LIKE OSCILLATIONS}

We want to determine the particle distribution in the configuration space when in the (33) the self-interaction term (wake potential) is negligible compared to the harmonic one. This means that the bunch is not dense enough to introduce appreciable effects of self-interaction. So that, (33) reduces to:

$$
i \tilde{\epsilon} \frac{\partial \Psi}{\partial s}=-\frac{\tilde{\epsilon}^{2}}{2} \frac{\partial^{2} \Psi}{\partial x^{2}}+\frac{1}{2} K_{p} x^{2} \Psi
$$

A complete set of modes associated to (34) is :

$$
\Psi_{m}(x, s)=\frac{\exp \left[-\frac{x^{2}}{4 \sigma^{2}(s)}\right]}{\left[2 \pi \sigma^{2}(s)\right]^{1 / 4}} H_{m}\left(\frac{x}{\sqrt{2} \sigma(s)}\right) \exp \left[i \frac{x^{2}}{2 \tilde{\epsilon} \rho(s)}+i(1+2 m) \Phi(s)\right] .
$$

In (35) the functions $\sigma(s), \rho(s)$ and $\Phi(s)$ are solutions of the following system of differential equations

$$
\begin{gathered}
\frac{d^{2} \sigma}{d s^{2}}+K_{p} \sigma-\frac{\tilde{\epsilon}^{2}}{4 \sigma^{3}}=0 \\
\frac{1}{\rho}=\frac{1}{\sigma} \frac{d \sigma}{d s} \\
\frac{d \Phi}{d s}=-\frac{\tilde{\epsilon}}{4 \sigma^{2}}
\end{gathered}
$$

and $H_{m}(x)$ are the Hermite-polynomials with $m$ a non- negative integer. Note that $\left|\Psi_{m}\right|^{2}$ for $m=0$ (fundamental mode) gives a Gaussian particle distribution. Remarkably, it can be easily proved that (36) is completely equivalent to

$$
\frac{d^{2} \sigma^{2}}{d s^{2}}+4 K_{p} \sigma^{2}=4 \mathcal{E}
$$

where

$$
\mathcal{E}=\frac{1}{2}\left(\frac{d \sigma}{d s}\right)^{2}+\frac{\tilde{\epsilon}^{2}}{8 \sigma^{2}}+\frac{1}{2} K_{p} \sigma^{2}=\text { const. , }
$$

Note that it is easy to recognize that: (a) $\sigma$ can be defined also in terms of the following quantum-like rms of $x$ (bunch length), namely

$$
\sigma(s)=\left[\int_{-\infty}^{\infty} x^{2}\left|\Psi_{0}(x, s)\right|^{2} d x\right]^{1 / 2}
$$

(b) equation for $\sigma(s),(39)$, i.e. (36), describes synchrotron-like oscillations; (c) the quantity $\frac{\sigma_{p}^{2}}{2 \gamma^{4}} \equiv \frac{1}{2}\left(\frac{d \sigma}{d s}\right)^{2}+\frac{\epsilon^{2}}{8 \sigma^{2}}$ appearing in (40) can be also introduced in terms of the following quantum-like definition of the rms linear momentum (bunch momentum spread):

$$
\sigma_{p}=\left[\epsilon^{2} \int_{-\infty}^{\infty}\left|\partial \Psi_{0} / \partial x\right|^{2} d x\right]^{1 / 2} ;
$$


(d) the quantity $\mathcal{E}$ can be also given by the following quantum-like definition (total averaged energy associated with the longitudinal bunch motion):

$$
\mathcal{E} \equiv \int_{-\infty}^{\infty} \Psi_{0}^{*} \hat{H} \Psi_{0} d x
$$

The equilibrium condition $d^{2} \sigma / d s^{2}=0$ gives, from (36)

$$
\sigma_{0}=\frac{1}{\gamma^{2} \sqrt{\left|K_{p}\right|}} \sigma_{p 0}
$$

where $\sigma_{0}$ and $\sigma_{p 0}$ are the equilibrium value of $\sigma$ and $\sigma_{p}$. Since for the present case the bwf is Gaussian, in obtaining (44) we have introduced the minimum value $\sigma_{0} \sigma_{p 0}=\epsilon / 2$ of the product $\sigma \sigma_{p}$ consistently with the following quantum-like uncertainty principle which holds in the framework of the thermal wave model :

$$
\sigma \sigma_{p} \geq \frac{\epsilon}{2} .
$$

\section{(B). ENVELOPE EQUATION AND BUNCH LENGTHENING}

Now we want to find an aberrationless solution of (33) following the standard techniques of nonlinear e.m. wave optics [20]. We look for a solution of (33) in the form:

$$
\Psi(x, s)=\frac{e^{-\frac{x^{2}}{4 \sigma(s)}}}{\sqrt[4]{2 \pi \sigma^{2}(s)}} e^{i \theta(x, s)}
$$

where the eikonal has been supposed as:

$$
\theta(x, s)=\frac{x^{2}}{2 \tilde{\epsilon} \rho(s)}+\Phi(s) .
$$

By substituting (46) and (47), separating the real part from the imaginary one, and expanding $|\Psi|^{2}$ up to the second-power of $x$ (aberrationless approximation) we get a coupled equation system for the effective particle bunch length $\sigma(s)$, the curvature radius of the wavefront $\rho(s)$, and the phase $\Phi(s)$. Therefore :

$$
\frac{d^{2} \sigma}{d s^{2}}+K_{p} \sigma-\frac{\xi}{\sigma^{2}}-\frac{\epsilon^{2}}{4 \gamma^{4} \sigma^{3}}=0
$$

where $\xi \equiv \frac{q^{2}}{e^{2}} \frac{n_{b}}{\sqrt{2 \pi} \gamma^{3} n_{p}}>0$. We first observe that in the limit of negligible self interaction $(\xi \approx 0),(48)$ gives the envelope equation for the synchrotron-like motion (see Eq. (36) ). Let us consider now the equilibrium condition, namely $d^{2} \sigma / d s^{2}=0$, when the self interaction is not negligible. In this case (48) gives:

$$
\alpha\left(\frac{\sigma}{\sigma_{0}}\right)^{4}-\delta\left(\frac{\sigma}{\sigma_{0}}\right)-1=0
$$

where $\alpha \equiv K_{p} /\left|K_{p}\right|$ and $\delta \equiv \xi /\left(\left|K_{p}\right| \sigma_{0}^{3}\right)$. Since $\sigma(s)$ is a positive function and $\xi$ is a positive constant, it can be easily seen that (49) is possible only for $\alpha=1$. Consequently, 
we conclude that the equilibrium solutions $\sigma_{0}^{\prime}$ associated with (48) exist only for $K_{p}>0$, namely $\phi_{s}=(2 n+1) \pi$, and satisfy the condition

$$
\sigma_{0}^{\prime}>\sigma_{0}
$$

This means that the competition between the effect on the particles due to the plasma wave potential well and the self interaction produces, in correspondence with the synchronous phase, a new equilibrium condition which results in a bunch lengthening according to (50). For $\delta<<1$ and $\alpha=1$ the solution of (49) is approximately $\sigma_{0}^{\prime} \approx(1+\delta / 4) \sigma_{0}$, but, as the self-interaction becomes more and more important, the bunch lengthening becomes more and more significant. For example, for $\delta=1, \sigma_{0}^{\prime} \approx 1.22 \sigma_{0}$, for $\delta=10$, $\sigma_{0}^{\prime} \approx 2.19 \sigma_{0}$, and for $\delta=100, \sigma_{0}^{\prime} \approx 4.64 \sigma_{0}$.

\section{(C). MODULATIONAL INSTABILITY}

We now analyze the response of the system to a small perturbation in the bunch distribution under the action of the self-interaction only (the plasma wave is considered switched off). In this case (33) assumes the following form

$$
i \frac{\partial \Psi}{\partial s}+P \frac{\partial^{2} \Psi}{\partial x^{2}}+Q|\Psi|^{2} \Psi=0
$$

For this generic cubic-NLS a small perturbation is unstable (stable) if the following condition (Lighthill criterion) is satisfied [19],[20] :

$$
P Q>0 \quad(P Q<0) .
$$

Consequently, for the (51) with $P=\tilde{\epsilon} / 2$ and $Q=q^{2} n_{b} /\left(e^{2} n_{p} \gamma^{3} \tilde{\epsilon}\right)$ we have only instability with respect to a small density perturbation. This is formally identical to the so-called modulational instability of an e.m. wave packet travelling in a nonlinear medium [19],[20] and for the present case it describes the self-bunching of the particle bunch under the action of its plasma wake potential.

(D). SOLITONS

Still considering the plasma wave switched off, a solitary solution of $(51)$ with $P=\tilde{\epsilon} / 2$ and $Q=q^{2} n_{b} /\left(e^{2} n_{p} \gamma^{3} \tilde{\epsilon}\right)$ is found by looking for a solution of the following envelope form:

$$
\Psi(x, s)=G\left(x-\beta_{0} s\right) e^{i k_{0} x-i \omega_{0} s}
$$

with $k_{0}$ and $\omega_{0}$ real numbers. According to the general theory of NLS equation [19], the condition $P Q>0$, satisfied by $(51)$, is the right condition to have solitons. The soliton-like solution for the beam density, which satisfies (3) and (4) is thus:

$$
\rho_{b}(x, s)=\frac{q^{2}}{e^{2}} \frac{\gamma n_{b}^{2}}{4 n_{p} \epsilon^{2}} \operatorname{sech}^{2}\left[\frac{q^{2}}{e^{2}} \frac{\gamma n_{b}}{2 n_{p} \epsilon^{2}}\left(x-\beta_{0} s\right)\right]
$$

$k_{0}=\frac{\gamma^{2} \beta_{0}}{\epsilon}$

$\omega_{0}=\frac{\epsilon}{2 \gamma^{2}} k_{0}^{2}-\left(\frac{q^{2}}{e^{2}} \frac{\gamma n_{b}}{2 n_{p} \epsilon^{2}}\right)^{2}$. 


\section{Remarks and conclusions}

In this paper we have presented a first review of novel approach for considering self-consistent interaction between the wakefield and the driving relativistic electron (positron) beam in a unmagnetized, overdense, collisionless plasma. For our purpose, we have employed TWM [3].

For describing transverse dynamics, the fluid equations have been used to study the wakefield dynamics in the presence of the transverse profile of the beam density. We thus have a selfconsistent coupling between the driving beam and the wakefields, and our treatment represents an improvement of a previous approach [1], which has completely ignored the reaction of the wakefield on the driver and did not consider the spatial evolution of the beam. It is found that the present model is capable of reproducing the main results found in the conventional theory of the beam self-interaction.

Furthermore, for the longitudinal dynamics we have described the nonlinear longitudinal dynamics of a relativistic charged particle bunch in cold, unmagnetized plasmas within the context of TWM [3]. We have shown that the nonlinear interaction between the bunch and the surroundings (plasma wave potential well and plasma wake fields) is governed by an appropriate NLS equation (equation (33) ), fully similar to that one which holds for the propagation of an e.m. bunch in a nonlinear medium in paraxial approximation. In this analogy, our system satisfies Lighthill criterion (modulational instability [19],[20]), and the density can assume a soliton-like profile. Physically, a sort of competition between the diffractive energy (i.e. thermal energy) and the self energy is established. We have instability when the self energy term overcomes the diffraction one. According to subsection 3.2 a soliton formation would be the natural evolution of the initial beam density modulation toward a self bunching which asymptotically gives a soliton-like envelope wave train. We finally observe that the present treatment has allowed us to describe the phase-slip oscillations (synchrotron-like oscillations) of the particle bunching in the plasma wave potential well as well as to obtain a longitudinal envelope equation including self-interaction.

\section{References}

[1] P. Chen, J. M. Dawson, R. W. Huff, and T. Katsouleas, Phys. Rev. Lett. 54, 693 (1985).

[2] J. B. Rosenzweig et al., Phys. Rev. Lett. 61, 98 (1988); H. Nakanishi et al., Phys. Rev. Lett. 66, 1870 (1991).

[3] R. Fedele and G. Miele, Nuovo Cimento D 13, (1991) 1527.

[4] R. Fedele and P.K. Shukla, Phys. Rev. A 44, (1992) 4045.

[5] R. Fedele, L. Palumbo, and V.G. Vaccaro, Proc. EPAC 92 (Berlin, 24-28 March 1992) H. Henke, H. Homeyer and Ch.Petit-Jean-Genaz Ed.s, Editions Frontieres (1992) 762.

[6] R. Fedele, G. Miele, L. Palumbo, and V.G. Vaccaro, Phys. Lett. A 179, (1993) 407.

[7] R. Fedele and V.G. Vaccaro, Physica Scripta T52, 36 (1994).

[8] R. Fedele and G. Miele, Phys. Rev. A 46, (1992) 6634.

[9] R. Fedele, F. Galluccio, and G. Miele, Phys. Lett. A 185, (1994) 93.

[10] R. Fedele, G. Miele, and F. Galluccio, INFN/TC-93/12, published in Proc. 1993 Particle Accelerator Conference. Washington D.C., 17-20 May 1993, p.209 (1993).

[11] R. Fedele, G. Miele and L. Palumbo, Phys. Lett. A 194, 113 (1994).

[12] J.J. Su, T. Katsouleas, J.M. Dawson, P. Chen, M. Jones, and R. Keinigs, IEEE Trans. Plasma Science PS-15, 192 (1987).

[13] W.H. Bennett, Phys. Rev. 45, 89 (1934) 
[14] J. D. Lawson, Particle Beam and Plasmas, CERN Rep.76-09, 13 May 1976, Lectures given in the Academic Training Programme of CERN 1973-1974, edited by A. Hofmann and E. Messerschmidt, Geneva(1976).

[15] R. Fedele and E.J.N. Wilson Nuovo Cimento D12, 1497 (1990).

[16] M. Sands The Physics of Electron Storage Rings, SLAC Report 121 (1971).

[17] J. Lawson, The Physics of Charged Particle Beams, 2nd ed. (Clarendon, Oxford, 1988).

[18] B. Zotter, Collective Effects - General Description, CAS Proc., CERN 85-19, November 1985, pp.415-431.

[19] G.B. Whitham, Linear and Nonlinear Waves, (J. Wiley, New York, 1974).

[20] S.A. Akhmanov, A.P. Sukhuorukov, and R.V. Khokhlov, Sov.Phys.Usp., 93609 (1968). 